\title{
Correlative Microscopy Study of FIB Patterned Stainless Steel Surfaces as Novel Nano- Structured Stents for Cardiovascular Applications
}

Michael Schmidt ${ }^{1}$, Feroze Nazneen ${ }^{2}$, Gregoire Herzog ${ }^{2}$, Damien Arrigan ${ }^{2}$, Paul Galvin ${ }^{2}$, Calum Dickinson $^{3}$, Johann P de Silva ${ }^{4}$, Declan Scanlan ${ }^{5}$, Neal O'Hara ${ }^{5}$, Graham L W Cross ${ }^{4}$, Nikolay Petkov $^{1}$ and Justin D Holmes ${ }^{1}$

${ }^{1}$ Electron Microscopy and Analysis Facility (EMAF), Materials Chemistry and Analysis Group, and

${ }^{2}$ Life Sciences Interface Group, Tyndall National Institute, Lee Maltings, University College Cork, Cork, Ireland

${ }^{3}$ MSSI Materials \& Surface Science Institute, University of Limerick, Limerick, Ireland ${ }^{4}$ CRANN Nanoscience Institute \& School of Physics, Trinity College, Dublin 2, Ireland ${ }^{5}$ Adama Innovations, CRANN, Trinity College Dublin, Dublin 2, Ireland

\begin{abstract}
Coronary artery disease is a major problem worldwide causing 7.2 million deaths worldwide annually, resulting from vascular occlusion, myocardial infarction and its complications. Stent implantation is a percutaneous interventional procedure that mitigates vessel stenosis, providing mechanical support within the artery. However, stenting causes physical damage to the arterial wall. It is well accepted that a valuable route to reduce in-stent re-stenosis can be based on promoting cell response to nano-structured stainless steel (SS) surfaces such as, for example, by patterning nano-pits in SS. In this regard patterning by Focussed Ion-Beam (FIB) milling offers several advantages for flexible prototyping (i) practically any substrate material that is able to withstand high vacuum conditions of the microscope chamber can be used, (ii) there is high flexibility in the obtainable shapes and geometries by modulating the ion beam current and the patterning conditions, (iii) reduced complexity of the pattering process e.g. it is a single-step process with a possibility of real-time monitoring of the milling progression. On the other hand FIB patterning of polycrystalline metals is greatly influenced by channelling effects and redeposition. Correlative microscopy methods present an opportunity to study such effects comprehensively and derive structure-property understanding that is important for developing improved pattering. In this report we present a FIB patterning protocol for nano-structuring features (concaves) ordered in rectangular arrays on pre-polished 316L Stainless Steel (SS) surfaces. An investigation based on correlative microscopy approach of the size, shape and depth of the developed arrays in relation to the crystal orientation of the underlying SS domains, is presented. The correlative microscopy protocol is based on cross-correlation of top-view Scanning Electron Microscopy (SEM), Electron Backscattered Diffraction (EBSD), and Atomic Force Microscopy (AFM).Various dose tests were performed, aiming at improved productivity by preserving nano-size accuracy of the patterned process. The optimal FIB patterning conditions for achieving reasonably high throughput (patterned rate of about $0.03 \mathrm{~mm}^{2}$ per hour) and nano-size accuracy in dimensions and shapes of the features, are discussed as well.
\end{abstract}

\section{INTRODUCTION}

Coronary artery disease causes 7.2 million deaths worldwide annually [1], resulting from vascular occlusion, myocardial infarction and its complications. Stent implantation is a 
percutaneous interventional procedure that mitigates vessel stenosis, providing mechanical support within the artery. However, stenting causes physical damage to the arterial wall, leading to inflammation, thrombosis (clotting), and neo-intimal hyperplasia (re-stenosis) [2]. Further improvements in next generation stent coating are needed to enhance the attachment and survival of endothelial cells on the stent surface. The research presented here develops novel nanostructured features on 316L Stainless Steel (SS) stent surfaces to promote rapid endothelial cell adhesion to reduce instent re-stenosis. Based on a literature survey, promising cell responses to nano-structured surfaces were identified including nano-pit features [3].

Conventional techniques for topographical surface modification or surface patterning at the nanoscale are lithography based techniques. In technologies typically derived from microelectronics, such as in X-ray lithography or UV lithography, patterns are formed by using a mask [4]. Such lithography processes are time-consuming with many steps, and inherently inappropriate for prototype designs and processes. Electron beam lithography, and lithography based on scanning tunnelling microscopy or atomic force microscopy are high-resolution mask less procedures, but with a very low throughput and unsuitable for wide surface nano-patterning [5]. Imprint lithography is a high resolution technique for nano-patterning of large surfaces, but it requires moulds and is restricted to polymeric materials which could then be used as etch masks [6]. Patterning by Focussed Ion-Beam (FIB) milling offers several advantages for flexible prototyping (i) practically any substrate material that is able to withstand high vacuum conditions of the microscope chamber can be used, (ii) there is high flexibility in the obtainable shapes and geometries by modulating the ion beam current and the patterning conditions, (iii) reduced complexity of the pattering process e.g. it is a single-step process with a possibility of real-time monitoring of the milling progression. Thus for any particulate type of substrate various depths as well as lateral dimensions including the optimal feature size can be obtained at minimum number of processing steps. On the other hand FIB patterning of polycrystalline metals is greatly influenced by channelling effects and re-deposition. Correlative microscopy methods present an opportunity to study such effects comprehensively and derive structure-property understanding that is important for developing improved pattering. In this report we present a FIB patterning protocol for nano-structuring features (concaves) ordered in rectangular arrays on pre-polished 316L Stainless Steel (SS) surfaces. An investigation based on correlative microscopy approach of the size, shape and depth of the developed arrays in relation to the crystal orientation of the underlying SS domains, is presented. The correlative microscopy protocol is based on crosscorrelation of top-view Scanning Electron Microscopy (SEM), Electron Backscattered Diffraction (EBSD), and Atomic Force Microscopy (AFM).Various dose tests were performed, aiming at improved productivity by preserving nano-size accuracy of the patterned process. The optimal FIB patterning conditions for achieving reasonably high throughput (patterned rate of

about $0.03 \mathrm{~mm}^{2}$ per hour) and nano-size accuracy in dimensions and shapes of the features, are discussed as well.

\section{EXPERIMENT}

\section{Focussed Ion Beam (FIB) patterning}

The FIB system used in the current study is the FEI Helios NanoLab 600i, which is a Dual beam FIB for localized milling and deposition, transmitting a $30 \mathrm{keV}$ beam of Ga+ ions combined with a high resolution scanning electron microscope (SEM). The resolution of FIB 
milling is approximately the same as that of the "spot" size (i.e., the diameter of the beam at the sample surface). In our process, tuning the working current from $0.28 \mathrm{nA}$ up to $0.92 \mathrm{nA}$, the feature size on the surface of the 316L SS stent surfaces changes from $120 \mathrm{~nm}$ up to $180 \mathrm{~nm}$.

Electropolished 316L steel substrates were used for this study. Prior to nano-structuring, the polished specimens were cleaned in acetone, ethanol, and finally in ultrapure water via an ultrasonic treatment for $10 \mathrm{~min}$. Nano-structured features (concaves) ordered in rectangular arrays were patterned on 316L steel surfaces using FEI Helios NanoLab 600i system. This system was used because of high beam quality and stage stability. Concave structures $180 \mathrm{~nm}$ diameters with pitch of $360 \mathrm{~nm}$, and targeted depths of $50-100 \mathrm{~nm}$ were fabricated on electropolished steel surfaces on areas of $250 \mu \mathrm{m} \times 200 \mu \mathrm{m}$. The concave arrays were fabricated at $30 \mathrm{keV}$ at $0.92 \mathrm{nA}$. For ease of referencing to certain locations the area was divided in squares of $9 \mu \mathrm{m} \times 9 \mu \mathrm{m}$ with $1 \mu \mathrm{m}$ distance to the next square and labelled in $\mathrm{X}$ and $\mathrm{Y}$ direction alphanumerically.

\section{Scanning Electron Microscopy (SEM) and Electron Backscattering Diffraction (EBSD)}

Scanning electron microscope (SEM) was used to analyze the topography of nano textured surfaces of the polished and patterned stainless steel. The SEM images presented were obtained using the SEM at the FEI Helios NanoLab 600i at an electron beam current of $5 \mathrm{kV}$ and $86 \mathrm{pA}$ beam current.

Electron backscattering diffraction (EBSD) was performed in a Hitachi SEM SU-70 equipped with an Oxford Instruments EBSD attachment AztecHKL at 10kV under 70 deg tilt angle and step size $2 \mu \mathrm{m}$.

\section{Atomic Force Microscopy (AFM) imaging.}

Atomic (scanning) force microscopy is performed utilizing a DME DS95-50 scan head and C21 controller. We use in-house fabricate diamond pillar probes of $27 \mathrm{~nm}$ diameter (Adama Innovations Ltd.). Samples are mounted on a high-stiffness X-Y tilt stage and levelled such that feature sidewalls are parallel to the columnar probe when scanning perpendicular to the cantilever. Acquisition parameters for non-contact mode scanning are optimised to facilitate optimal tracking of high-aspect ratio features.

\section{RESULTS AND DISCUSSION}

The size, shape and depth of the pits is dependent on the surface sputtering caused by Ga-ion bombardment. During ion-beam bombardment surface atoms are removed from the SS target by creating recoil cascades that come back of the target, and which give surface atoms enough energy so that they are driven away from the target. The recoil events within a specific material are ruled out by the ion-beam energy, inclination towards the beam and most importantly the chemical composition and crystal structure of the target material. From the polycrystalline nature of the SS stent material one can assume that when subjected to ion milling or imaging it will show pronounced channelling contrast. It is well known for $\mathrm{Si}$ or $\mathrm{Cu}$ that it etches and mills faster in preferred directions [7-9]. Similarly polycrystalline metals will show milling rates that are varying by the different orientation of grains towards the incoming beam. In order to determine a correlation between crystal grain orientation on one hand and shape, size and depth of the FIB milled concaves on the other hand, EBSD mapping was accomplished before the area was patterned. Figure 1 shows the random size and orientation of the crystal grains. 
Figure 2 illustrates how much this anisotropic milling affects the desired outcome of uniform concaves. In general the intensity of the emitted SE depends on the different inclination of the sample surface towards the incoming beam. Thus, the grey levels in the SEM image are directly linked to the surface topography, e.g. the shape of the pits and side-wall profile. In this way we can correlate the EBSD data to the grey levels in the SEM images. Shown in Figure 2 are examples from the bottom right hand corner of the EBSD mapped area. The detail view in figure $2 \mathrm{~b}$ shows a very good uniformity of the holes.
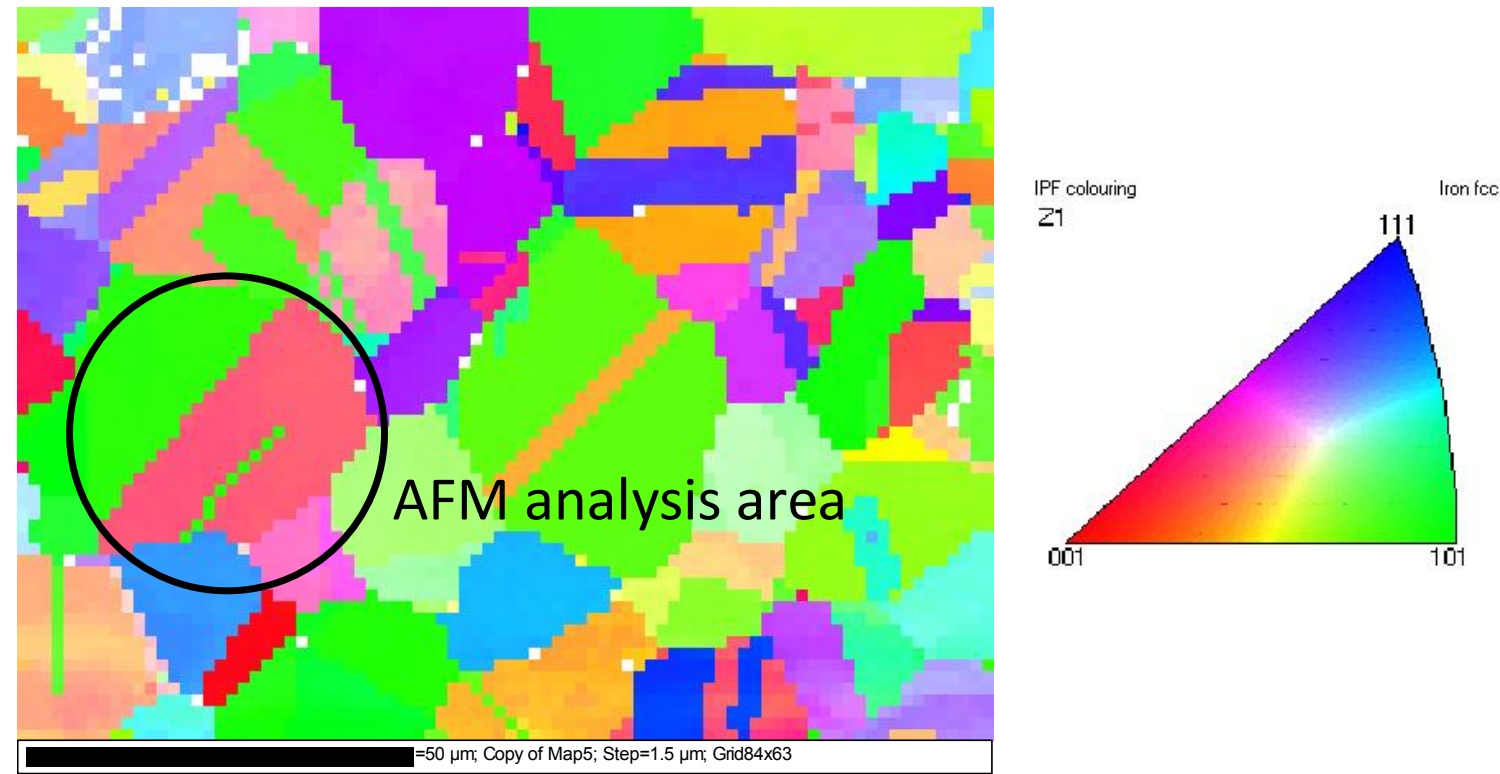

Figure 1. Inverse pole figure (IPF, Z-map) from the EBSD analysis, the highlighted area is analysed by AFM.
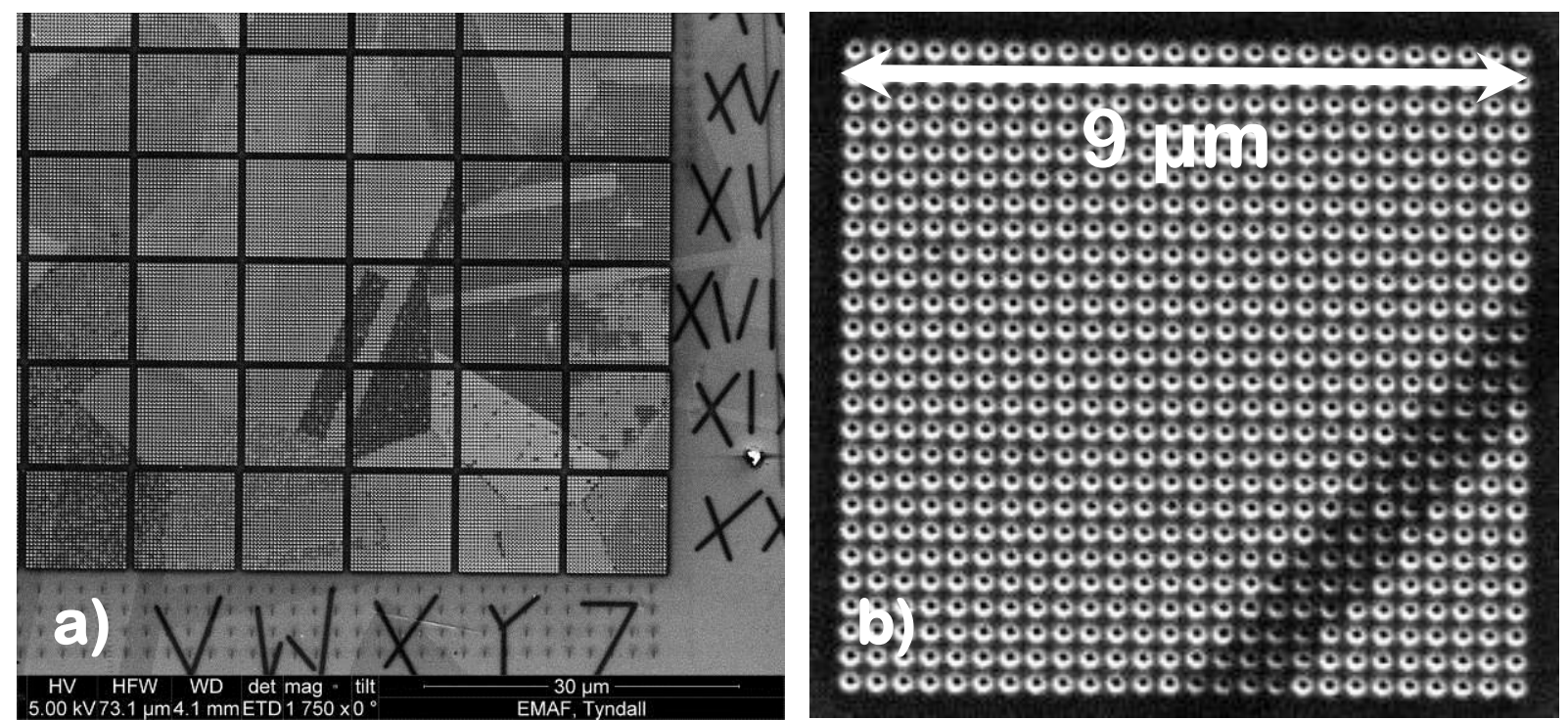

Figure 2. SEM images after FIB patterning. a) The marks from the EBSD analysis are still visible at the bottom of the image as dark dots in a $2 \mu \mathrm{m} \times 2 \mu \mathrm{m}$ steps array. b) Detail view illustrates the very good uniformity of the holes. 

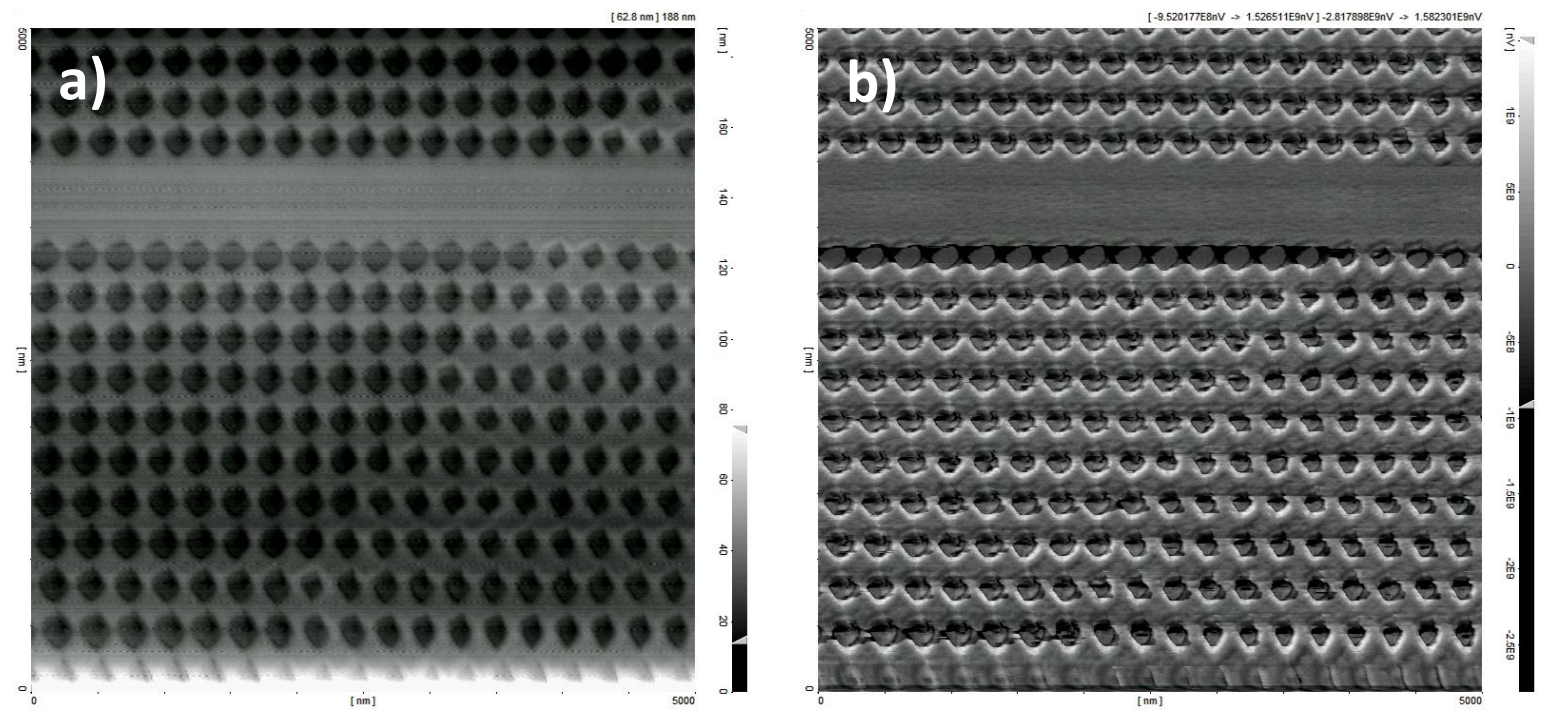

Figure 3. AFM detail analysis of shape and size of holes. a) Trace b) Phase. Very obvious are the different shaped holes in the differently oriented grains.

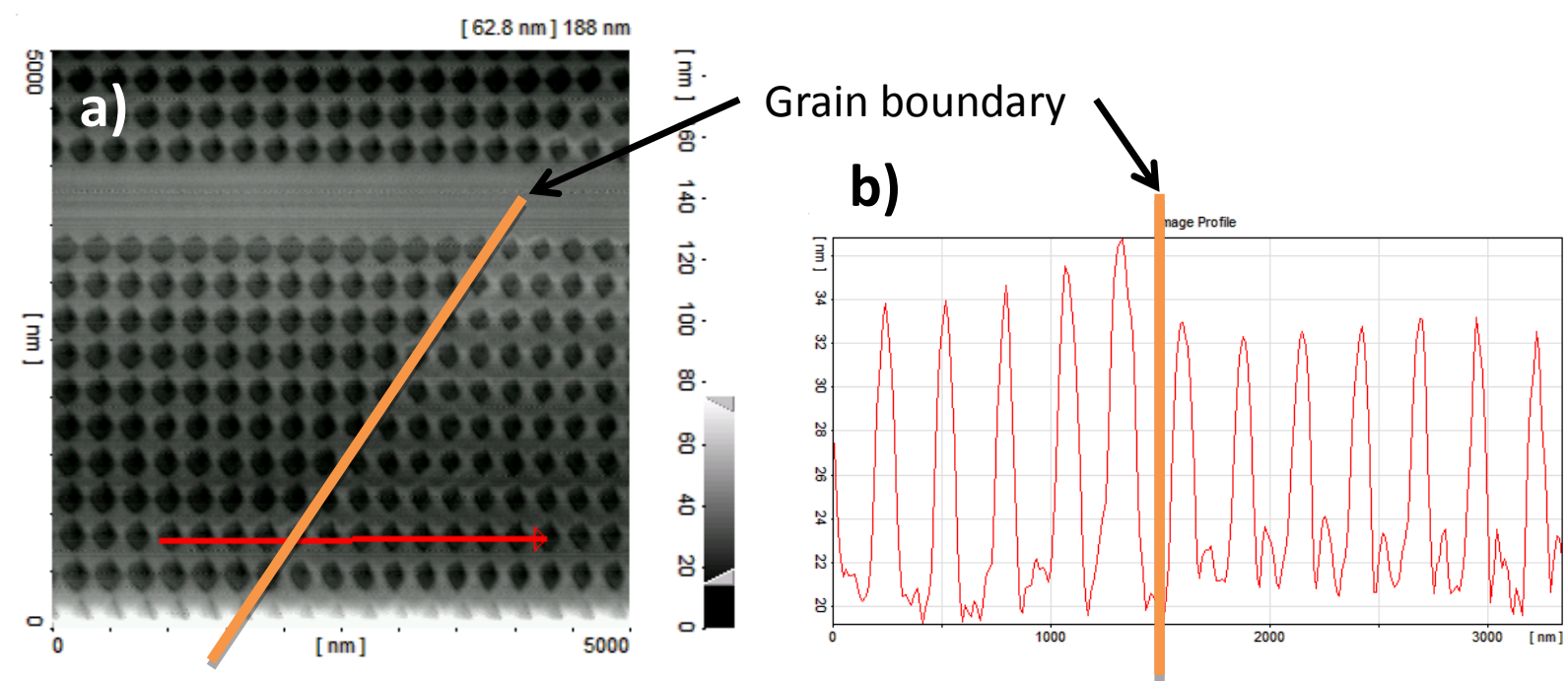

Figure 4. AFM grain boundary analysis. a) topography map of the marked region from figure 1 across the big grain boundary. b) Depth profile across the grain boundary along the arrow marked in a)

The size, shape and depth of the patterned structure was further analysed by AFM (figures 3 and 4) with newly developed high aspect ratio diamond tips. The use of the in-house developed diamond pillars as AFM tips has the advantage of much improved lateral and sidewall tracking, accompanied by significantly increased depth profiling. While in the SEM the holes looked very uniform and circular regardless of their depth, the AFM reveals the shape of the holes actually as irregular (figure 3). The structures that appear in the SEM with the brightest contrast showed deeper and sharper edges than the structures that appear darker in SEM contrast. AFM topography scans (figure $3 \mathrm{a}$ ) show that the brighter contrast is related to deeper holes. The phase scans (figure $3 b$ ) confirm that the brighter areas with the deeper holes show a greater phase shift that can be related to the different mechanical properties of the individual grains. The dependency of shape, size and depth of the milled pits from the crystallographic orientation of 
the SS grain can be observed in figure 4, though not as pronounced as the SEM images had hoped for. While in the SEM image there is a sharp contrast between the two crystal phases there is actually only about $2 \mathrm{~nm}$ difference in depth. Overall seem the pits very shallow in this region with only 10-16nm depth. From the profile (figure 4b) can be concluded that there is a peak in the middle of the pits at the bottom. This was confirmed by scanning in perpendicular direction so that both sides of the tips were used to exclude a measuring artefact. A very possible explanation for this peak is that the ion beam was over-focused during the milling of the holes which results in a ring structure instead of Gaussian distribution across the beam diameter. As the beam energy is dispersed over a bigger area the resulting depth is smaller. This illustrates also very nicely the limits of the patterning with FIB, even if the beam is circular and the SEM and FIB images show very uniform circular holes, the AFM analysis with the high aspect ratio diamond pillars reveal that the pits are neither uniform nor have a Gaussian depth profile. Further AFM analysis will be necessary in other regions across grain boundaries to relate the size, shape and depth to the crystal orientations.

\section{CONCLUSIONS}

FIB has compelling advantages for flexible prototyping compared to other traditional techniques, however the milling rates and the corresponding shape and size of the formed structures is largely affected by the grain size of the polycrystalline stainless steel. Moreover this method is limited to about $120 \mathrm{~nm}$ resolution for the desired concave depth and uniform scan size of $200 \mu \mathrm{m}$ x $200 \mu \mathrm{m}$ at reasonable writing times. Nevertheless the formed structures show large variation of concave size, shape and depths and as such might serve as a resourceful platform for screening large variations of cell/pattern SS stent interactions.

\section{ACKNOWLEDGMENTS}

This work was supported through 09/SIRG/I1621, NBIPI, and INSPIRE initiatives funded by the Irish Government PRTLI 4, NDP 2007-2013.

\section{REFERENCES}

1.http://www.who.int/mediacentre/factsheets/fs317/en (2011)

2. Lowe H C, Oesterle S N, and Khachigian L M J. Am. Coll. Cardiol 39 183-193 (2002)

3. Dalby M J, Gadegaard N, and Wilkinson, C D W J Biomed Mater Res Part A 4 973-979 (2008)

4. Spille E and Feder R Topics in Applied Physics Volume 22 (Berlin: Springer) pp35-92 (1977)

5. Vieu C, Carcenac F, Pépin A, Chen Y, Mejias M, Lebib A, Manin-Ferlazzo L, Couraud L, Launois H Appl. Surface Sci. 164111 (2000)

6. Chou Y S, Krauss P R, and Renstrom P J Appl. Phys. Lett. 673114 (1995)

7. Bean K E IEEE Transactions on Electronic Devices 25 1185-1193 (1978)

8. Seidel H, Csepregi L, Heuberger A, Baumgärtel H J Electrochem Soc 1373612 (1990)

9. Kempshall B W, Schwarz S M, Prenitzer B I, Giannuzzi L A, Irwin R B, Stevie F A J Vac Sci Technol B 19 749-754 (2001) 